\title{
History of Chlamydia Trachomatis Infection Threatens Fertility in Women with Unilateral Tubal Occlusion
}

\author{
Satoko Goa $^{1,2}$, Keiichi Kumasawa ${ }^{2,}$, , Masaaki Ono ${ }^{1}$, Takeshi Taniguchi ${ }^{1}$ \\ ${ }^{1}$ Department of Obstetrics and Gynecology, Taniguchi Hospital, Izumisano City, Japan \\ ${ }^{2}$ Department of Obstetrics and Gynecology, Osaka University, Suita City, Japan

\section{Email address} \\ ing31050805@yahoo.co.jp (S. Goa), kumasawa@gyne.med.osaka-u.ac.jp (K. Kumasawa), mono55max@hh.em-net.jp (M. Ono), \\ takeshi@taniguchi-hp.org (T. Taniguchi) \\ ${ }^{*}$ Corresponding author
}

\section{To cite this article:}

Satoko Goa, Keiichi Kumasawa, Masaaki Ono, Takeshi Taniguchi. History of Chlamydia Trachomatis Infection Threatens Fertility in Women with Unilateral Tubal Occlusion. Journal of Gynecology and Obstetrics. Vol. 4, No. 6, 2016, pp. 34-37. doi: $10.11648 /$ j.jgo.20160406.11

Received: August 18, 2016; Accepted: September 12, 2016; Published: October 8, 2016

\begin{abstract}
Tubal factors are associated with infertility. To evaluate the influence of past Chlamydial infections on female fertility with unilateral tubal occlusion, we compared the cumulative pregnancy rate of Chlamydia antibody seropositive women with those who are seronegative. A retrospective, case-controlled study was designed. A total of 54 consecutive infertile women with unilateral tubal occlusion diagnosed by hysterosalpingography (HSG) during January, 2009 to April, 2013 were enrolled in this study. Each patient was followed up for three years. The study group was composed of 19 Chlamydia antibody seropositive women. The control group consisted of 35 who are seronegative. The cumulative pregnancy rate of the study group was $6 / 19(31.5 \%)$ and that of the control group was $19 / 35(54.3 \%)$. This suggested that the patients with past Chlamydial infections had more difficulty becoming pregnant than those without prior infections. There were no significant differences of clinical parameters between the two groups. This study suggested that past Chlamydia infection may contribute to lower pregnancy rates in infertile women with unilateral tubal occlusion. Patients with both unilateral tubal occlusion and Chlamydia antibody seropositive may do better to proceed to in vitro fertilization (IVF).
\end{abstract}

Keywords: Chlamydia Trachomatis Infection, Unilateral Tubal Occlusion, In Vitro Fertilization (IVF)

\section{Introduction}

Tubal factors are estimated to be associated with infertility in approximately $40 \%$ and $85 \%$ of infertile women in developed and developing countries, respectively [1]. Salpingitis accounts for $50 \%$ of tubal factors causing infertility [2]. Furthermore, it has been reported that more than a half of tubal damage is attributed to Chlamydia infection [3].

Chlamydia trachomatis infection is the most common sexually transmitted bacterial infection worldwide. Untreated Chlamydia infections can lead to serious complications. Untreated Chlamydia infection in man can cause urethritis, epididymitis and proctitis. On the other hand, up to $30 \%$ of women with untreated Chlamydia infections developed pelvic inflammatory disease (PID). Of these women, the majority had symptoms that were too mild or nonspecific to cause them to seek medical treatment. Regardless of symptom intensity, the consequences of PID are severe [4].

If women with Chlamydia infection were left untreated, $20 \%$ of them become infertile, and $9 \%$ will be affected with life-threatening tubal pregnancy.

Chlamydia infection during pregnancy might lead to infant conjunctivitis and pneumonia and maternal postpartum endometritis. Additionally it is association with an increased risk for the transmission or acquisition of HIV and is also attributed to be a risk factor for the development of cervical carcinoma [4].

In addition to medical problems, Chlamydia infection also results in economic problems. In 2008 direct medical costs of 
Chlamydia infections in USA were estimated at $\$ 516.7$ million [4]. Early diagnosis and treatment of infected individuals is required to prevent the spread of the disease and severe sequelae.

It has been reported that there were no significant differences in cumulative pregnancy rates after 3 cycles of controlled ovarian hyperstimulation $(\mathrm{COH})$ and intrauterine insemination (IUI) between women with unilateral proximal tubal occlusion and those with bilateral normal tubes [5]. Other studies showed that there were no differences in pregnancy rates between Chlamydia IgG seronegative and seropositive women [6] [7] [8]. The management of infertile women with unilateral tubal occlusion differs between medical practitioners, but a serum Chlamydia antibody test plays an important role in deciding management of women with unilateral tubal occlusion. Some physicians adopt infertility treatments at an earlier step, such as timed intercourse. Others propose artificial reproductive technology from the beginning [9].

\section{Methods}

A retrospective case-controlled study was designed. A total of 54consecutive infertile women with unilateral tubal occlusion diagnosed by HSG during the period from January, 2009 to April, 2013 were enrolled in this study. The study group consisted of 19 Chlamydia antibody seropositive women, and the control group consisted of 35 seronegative women. HSG was performed with a water-soluble contrast media. Patients with uterus malformations were excluded. IVF treated patients were also excluded. They received infertility treatment by timed intercourse or intrauterine insemination (IUI) with or without ovulation inducing drugs. Treatment (recanalization, salpingoplasty) for the occluded fallopian tube was not provided. Past Chlamydia infection was diagnosed by the serum concentration of Chlamydia IgG or IgA antibodies. The Chlamydia IgG and IgA antibody assays were performed according to the manufacturers' instructions (LSI medience Corporation, Tokyo, Japan). The qualitative outcome of the assay (cut-off index) was categorized as negative $(<0.9 \mathrm{ng} / \mathrm{ml})$ or positive $(\geqq 0.9 \mathrm{ng} / \mathrm{ml})$. Diagnosis of clinical pregnancy was based on a positive urine or serum hCG test in association with an intrauterine gestational sac using ultrasonography. Five years of observation was performed from the first visit to our hospital. Statistical analysis was performed using SPSS version22 (IBM, Chicago, USA). Student- $t$ test was performed for analyzing the means and the chi-square test was adopted for the analysis of odds ratios. $\mathrm{P}<0.05$ was considered statistically significant.

\section{Results}

The cumulative pregnancy rate of the study group was $6 / 19(31.5 \%)$, and that of control group was $19 / 35(54.3 \%)$. (Odds ratio: 0/39:95\%, CI: 0.12-1.25).

Between the two groups, there is no significant difference of age, serum level of basal LH/FSH, body mass index (BMI), use of ovulation induction agent and previous pregnancy history. In this study male factors were not detected (Table1). Both median and mean of the duration until the pregnancy didn't differ between the two groups (Fig 1.). Chlamydia antibody seropositive women did not become pregnant after 13 cycles. On the other hand, the cumulative pregnancy rate of Chlamydia antibody seronegative group gradually increased with the prolongation of the treatment cycles (Fig1).

Table 1. Comparison of clinical parameters between Chlamydia seropositive and Seronegative groups.

\begin{tabular}{llll}
\hline & $\begin{array}{l}\text { Seropositive } \\
(\mathbf{N = 1 9 )}\end{array}$ & $\begin{array}{l}\text { Seronegative } \\
(\mathbf{N}=\mathbf{3 5})\end{array}$ & P value \\
\hline Age & $32.5 \pm 4.8$ & $33.8 \pm 4.8$ & 0.359 \\
Day3 FSH $(\mathrm{mIU} / \mathrm{ml})$ & $5.23 \pm 1.6$ & $7.4 \pm 2.8$ & 0.026 \\
Day3 LH $(\mathrm{mIU} / \mathrm{ml})$ & $4.67 \pm 2.4$ & $5.4 \pm 2.6$ & 0.426 \\
LH/FSH & $0.9 \pm 0.9$ & $0.8 \pm 0.5$ & 0.633 \\
Pregnant & 6 & 19 & 0.113 \\
Induction of Ovulation & 11 & 25 & 0.314 \\
Primary Infertility & 12 & 17 & 0.305 \\
BMI $\left(\mathrm{kg} / \mathrm{m}^{2}\right)$ & $20.4 \pm 3.3$ & $21.2 \pm 3.9$ & 0.494 \\
\hline
\end{tabular}

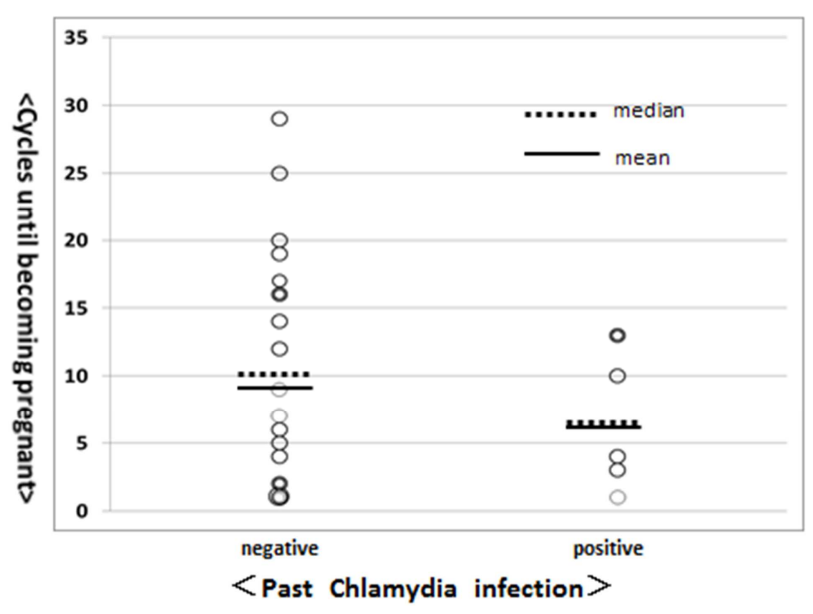

Figure 1. Past Chlamydia infetcions and cycles until pregnancy.

\section{Discussion}

HSG is one of the standard fertility work ups [10]. If bilateral tubes were found to be passed by HSG, laparoscopy can be delayed or refused because of the low risk of tubal occlusion. An HSG finding of bilateral tubal occlusion is an indication for either laparoscopy, tuboplasty or IVF. However, thus far, there is no standard management of unilateral tubal occlusion. There are several potential management strategies:

(1) further evaluation of the pelvis by laparoscopy,

(2) repeating the HSG with or without catheterization,

(3) attempting to achieve pregnancy with $\mathrm{COH}$ and IUI and

(4) referring the couple for IVF. One previous study reported that fecundity rate ratios were $0.81(95 \%$ confidence interval 0.47 to 1.4$)$ for a one-sided tubal occulusion and $0.30(95 \%$ confidence interval 0.13 to 0.71 ) for a two-sided occulusion, so one-sided tubal pathology detected by HSG has limited prognostic significance [11].

There was another report that 93 infertile women with tubal occlusion who had salpingostomy performed and a second 
look laparoscopy revealed that $45(48 \%)$ of them had evident fallopian tubes on at least one side. However, only $12(13 \%)$ had live births. This report suggest that patients with severe adhesions and poor tubal status should be primarily directed to IVF programs rather than to microsurgery [12].

The role of fallopian tubes in pregnancy is as follows. 1 . Capture and transfer of an ovulated ovum for fertilization at the ampula. 2. According to increasing estrogen, the ovum was retained at the ampulla by contraction of its smooth muscle. 3 . Forming an embryo growth environment by increasing progesterone action. Furthermore, patients of fimbria firmness syndrome and Kartagener's syndrome suffer from infertility. The mechanism of fallopian tube disorder caused by Chlamydia infection is considered to be as follows. At first, Chlamydia infection is established in the cervix by having sex with infected men. Next, it spreads to the endometrium, tube lining membrane and fimbriae of the tube neighborhood, pelviperitoneum, and liver lap. Only about $20 \%$ of patients suffering from Chlamydia infection are asymptomatic while others lack subjective symptoms which in turn leads to infection of subsequent sexual partners [13]. Long lasting fallopian tube inflammation causes the fimbriae cells to be destroyed and the function of capture and transformation of the fallopian tube is negatively affected. Next, subcutaneous interstitial fibrosis follows which leads to narrowing or occlusion of the tubal lumen. Therefore, contraction of smooth muscle in the fallopian tube is affected. Furthermore, extensive inflammation caused by Chlamydia infection activates host specific immune responses to Chlamydial antigens. One previous report stated that even after treatment for Chlamydia infection, immune responses continue due to cross reaction to Chlamydia heat shock protein (HSP) and human HSP [2]. The embryonic growth environment cannot be the same as it was prior to Chlamydia infection. Taking this into consideration, the embryo growth environment is not thought to be good even if the tubes are not occluded following the Chlamydia infection. In addition, the condition of tissues in the tubes and pelvis is not considered to be completely recovered if they have been exposed to inflammation even once [14]. Consequently, for infertile women Chlamydia infection screening is necessary to identify and treat this infection.

Farhi et al. reported that there were no significant differences in cumulative pregnancy rates after 3 cycles of controlled ovarian hyperstimulation $(\mathrm{COH})$ and intrauterine insemination (IUI) between women with unilateral proximal tubal occlusion and those with bilateral normal tubes [5]. On the other hand, our results suggest that Chlamydia antibody seropositive patient should be better to proceed to in vitro fertilization (IVF) before 6 or 7 cycles of IUI. Of course our results didn't conflict with Farhi's report.

In this study investigation a role for endometriosis or the occlusion location in the fallopian tube couldn't be performed. Accumulation and observation of patients for more detailed analysis will be needed.

The Centers for Disease Control and Prevention (CDC) recommends $1 \mathrm{~g}$ azithromycin orally in a single dose, or 100 $\mathrm{mg}$ doxycycline orally twice a day for seven days for uncomplicated genital-urinary infection. Alternate regimens include erythromycin $500 \mathrm{mg}$ orally four times a day or ofloxacin $300 \mathrm{mg}$ orally twice a day for seven days. Compared with the conventional therapy, azithromycin has advantage of having better compliance. All the other regimens have similar cure rates and adverse effect profiles [4].

Patients should be instructed to abstain from sexual intercourse for seven days after the treatment initiation, and the partners should be treated simultaneously in order to prevent re-infection. Recurrent Chlamydia infection increases the risk for developing ectopic pregnancy and PID [4].

Levofloxacin, ofloxacin and doxycycline are contraindicated during pregnancy. Therefore, azithromycin 1 g orally in a single dose or amoxycillin $500 \mathrm{mg}$ orally thrice a day is recommended. Amoxycillin is reported to be more effective and with fewer side effects than erythromycin in treating antenatal chlamydial infection. Testing for cure is indicated in patients who are pregnant and should be performed three weeks after completion of treatment. If the risk of re-exposure is high, screening should be repeated throughout pregnancy [4].

In the future vaccination could be substantially more effective than other biomedical interventions in controlling epidemics of Chlamydia infection [15].

Administrating a protective vaccine to adolescents before their first sexual experience may induce a significant reduction in prevalence which could not be obtained by screening teenagers [15]. However, the ultimate intervention the development of an effective vaccine is still far away and further research is required. The best available intervention today is the early detection by screening and treatment of infected cases and their sexual partners. Further research will be needed for prevention of Chlamydia infection.

\section{Conclusions}

Our study suggested that past Chlamydia infection may contribute to lower pregnancy rates in infertile women with unilateral tubal occlusion.

Our results indicate that when after about 6 cycles of timed intercourse or IUI, clinicians may do better to offer IVF treatment, because few patients with past infection conceived after 12 cycles (average 7.3 cycles). In this study, a role for endometriosis or the occlusion location in the fallopian tube was not investigated. Therefore, further observation will be necessary in future.

\section{References}

[1] Haggerty CL, Gottlieb SL, Taylor BD, Low N, Xu F, Ness RB. "Risk of sequelae after Chlamydia trachomatis genital infection in women" J Infect Dis. Vol S1, pp34-55. Jun 2010 .

[2] Mascellino MT, Boccia P, Oliva A. "Immunopathogenesis in Chlamydia trachomatis Infected Women." ISRN Obstet Gynecol, 436936, 2011. 
[3] Campbell LA, Patton DL, Moore DE, Cappucio AL. "Detection of Chlamydia trachomatis deoxyribonucleic acid in women with tubal infertility." Fertil Steril, Vol 59, pp 45-50, Jan 1993

[4] Centers for Disease Control (CDC) and Prevention, "Recommendations for the Laboratory-Based Detection of Chlamydia trachomatis and Neisseria gonorrhoeae." Vol 63 No2, March 2014.

[5] Farhi J, Ben-Haroush A, Lande Y, Fisch B. "Role of treatment with ovarian stimulation and intrauterine insemination in women with unilateral tubal occlusion diagnosed by hysterosalpingography." Fertil Steril, Vol 88, pp 396-400, August 2007.

[6] Idahl A, Boman J, Kumlin U, Olofsson JI. "Demonstration of Chlamydia. trachomatis IgG antibodies in the male partner of the infertile couple is correlated with a reduced likelihood of achieving pregnancy." Hum Reprod. Vol 19, No5, pp 1121-6, 2004.

[7] Denise A. M. Perquin, Matthias F. C. Beersma, Anton J.M.de Craen, Franz M. Helmerhorst, "The value of Chlamydia trachomatis-specific IgG antibody testing and hysterosalpingography for predicting tubal pathology and occurrence of pregnancy." Fertil Steril. Vol 88, pp 224-6, July 2007.

[8] Batool Hossein Rashidi, Leili Chamani-Tabriz, Fadieh Haghollahi et al. "Effects of Chlamydia trachomatis Infection on Fertility; A Case-Control Study.” J Reprod Infertile, Vol 14, No2, pp 67-72. Apr 2013.

[9] Martin D. Keltz, Puja Sharma Gera. "Chlamydia serology screening in infertility patients." Fertil Steril. Vol 85, pp 752-4, March 2006.
[10] Practice Committee of the American Society for Reproductive Medicine, Committee opinion "role of tubal surgery in the era of assisted reproductive technology." Fertil Steril. Vol 97, No 3, pp 539-545, Mar 2012.

[11] Broeze KA, Opmeer BC, Van Geloven N, Coppus SF, Collins JA, Den Hartog JE, Van der Linden PJ, Marianowski P, Ng EH, Van der Steeg JW, Steures P, Strandell A, Van der Veen F, Mol BW. "Are patient characteristics associated with the accuracy of hysterosalpingography in diagnosing tubal pathology? An individual patient data meta-analysis." Hum Reprod Update. Vol 17, No3 pp293-300, May-Jun 2011.

[12] T. J. Laatilainen, A. K, Tenhunen, P. K. Venesmaa, and D. L. Apter. "Factors Influencing the Success of Microsurgery for Distal Tubal Occlusion.” Gynecology and Obstetrics. Vol 243 No2, pp 101-106, 1988.

[13] Mårdh PA. "Tubal factor infertility, with special regard to chlamydial salpingitis." Curr Opin Infect Dis. Vol 17, No1, pp49-52, Feb 2004.

[14] Mirjana Kessler, Julia Zielecki, Oliver Thieck, et al. "Chlamydia trachomatis Disturbs Epithelial Tissue Homeostasis in Fallopian Tubes via Paracrine Wnt Signaling." Am J Pathol. Vol 180, No. 1, pp186-198, January 2012.

[15] Sam vasilevsky, Gillbert Greub, Denise Nardelli-Haefliger, David Baud "Genital Chlamydia trachomatis: Understanding the Roles of Innate and Adaptive Immunity in Vaccine Research" Clinical Microbiology Reviews Vol 27, No.2, pp 346-370, April 2014. 